\title{
Invasive Pneumonia with Skin Peeling caused by Group A Beta-Hemolytic Streptococcus and Kawasaki Disease
}

\author{
Meshal Almeshary ${ }^{1}$, Alanoud Abdullah Abuhaimed ${ }^{2}$, Dania Mohammed Alkelabi' ${ }^{2}$, Mohamad-Hani Temsah ${ }^{3}$ \\ 1- General Pediatric Unit, 2- College of Medicine, \\ 3- Pediatric Intensive Care Unit, King Saud University Medical City, Riyadh, Saudi Arabia \\ Corresponding author: Meshal Zaid Almeshary,Email: Malmeshary1@ksu.edu.sa,mobile:00966548453267
}

\begin{abstract}
Group A Streptococcus commonly causes cellulitis and pharyngitis, but in rare instances, it may cause pneumonia. Herein, we report a case of invasive pneumonia with classic symptoms of Kawasaki disease (KD). This case highlights the importance of considering KD in patients with pneumonia, especially when they present with typical KD features and do not respond to antibiotics.

Objectives: To present our case of invasive pneumonia with skin peeling caused by group a beta-hemolytic streptococcus and kawasaki disease presented to General Pediatric Unit, King Saud University Medical City, Riyadh, Saudi Arabia.

Subjects and Methods: Case study of a four-years-old boy who was presented to the hospital with fever, cough, and dyspnea that persisted for 7 days. His medical history was unremarkable, and his immunizations were up to date as per the Saudi Ministry of Health vaccination schedule for children of that age.

Results: As a result, the final diagnosis was confirmed as KD with GAS. Treatment with vancomycin and tazocin was sustained for a whole of three weeks, and subsequently the case was discharged after a followup cardiology examination. Echocardiography was repeated after six weeks of illness with no evidence of coronary dilatation.

Conclusion: A diagnosis of KD should be considered in patients with pneumonia who exhibit the characteristic symptoms of $\mathrm{KD}$, especially when a poor response to antibiotic treatment is observed.
\end{abstract}

Key words: Invasive pneumonia, Group A streptococcus, Kawasaki disease.

\section{INTRODUCTION}

Pneumonia is a common but serious infection affecting children worldwide ${ }^{(1)}$. The major bacterial pathogens of this disease are Streptococcus pneumoniae and Staphylococcus aureus ${ }^{(2)}$. Group A Streptococcus (GAS) typically causes cellulitis and pharyngitis ${ }^{(3)}$, and the lower respiratory tract is an uncommon site of GAS infections ${ }^{(4)}$. Kawasaki disease (KD) is an acute and selflimiting disease that results in medium-vessel vasculitis and mostly comprises the coronary arteries. The clinical manifestation of KD is exceedingly mutable, with no pathognomonic laboratory results.

\section{CASE PRESENTATION}

A four-year-old boy was brought to the hospital in January 2019 with fever, cough, and dyspnea that persisted for 7 days. His medical history was unremarkable, and his immunizations were up to date as per the Saudi Ministry of Health vaccination schedule for children of that age. On examination, he appeared ill with respiratory distress and had a respiratory rate of 50 breaths/min, while maintaining an oxygen saturation of $98 \%$ on room air. The patient was febrile $\left(39.3^{\circ} \mathrm{C}\right)$ with a heart rate of $158 / \mathrm{min}$ and a blood pressure of $103 / 66$
mmHg. His lung sounds were equal and bilaterally vesicular.

In addition, he had bilateral, non-exudative conjunctivitis, cracked lips, strawberry tongue, unilateral cervical lymphadenopathy measuring $1.5 \mathrm{~cm}$, erythematous macular rash in the truncal area and erythema of the hands and feet. Upon hospitalization, laboratory checks showed the next values: white blood cell (WBC) count of $13.9 / \mu \mathrm{l}$ (57\% neutrophils), hemoglobin level of $10 \mathrm{~g} / \mathrm{dl}$, platelet count of $135 / \mu \mathrm{l}$, Creactive protein level of $160 \mathrm{mg} / \mathrm{l}$, erythrocyte sedimentation rate of $104 \mathrm{~mm} /$ hour, and albumin level of $21 \mathrm{~g} / \mathrm{l}$. Urine analysis indicated $13 \mathrm{WBC} / \mathrm{HPF}$ with negative culture. The liver and renal function test findings were normal.

Several investigations including blood culture, mycoplasma IgM tests, and nasopharyngeal aspirates for virology tests such as $\mathrm{H} 1 \mathrm{~N} 1$ and MERS-CoV, were performed for detection of infectious. The results from these tests were all negative.

However, the result of rapid antigen detection test for GAS was positive, and the ASO titer was 1110 units $/ \mathrm{ml}$. Chest radiograph revealed multiple homogeneous circular opacities (Figure 1). 


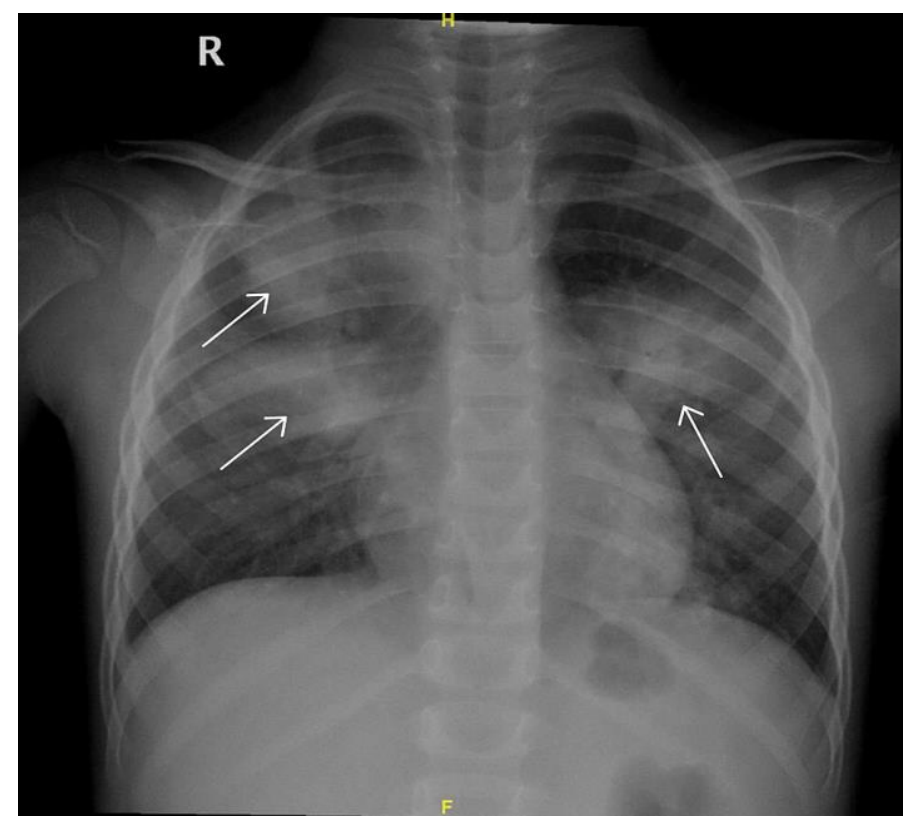

Figure (1): Chest radiograph showing multiple homogeneous circular opacities (arrows)

The patient was administered ceftriaxone and azithromycin for two days and received two doses of intravenous immune globulin (IVIG) along with low-dose aspirin (4.6 mg/kg/day). However, no resulting improvement was noted in the fever pattern. Subsequently the patient was administered vancomycin and tazocin to control persistent high-grade fever, as recommended by the infectious disease team. A differential diagnosis of complicated pneumonia was made. In the following days, the fever persisted with associated thrombocytosis (highest count was 842). Echocardiography was performed on the ninth day of illness, which showed normal function and anatomy of the heart with no dilatation of the coronary arteries. Contrast-enhanced chest computed tomography (CT) confirmed bilateral pneumonic consolidation with cavitation (Figure 2).

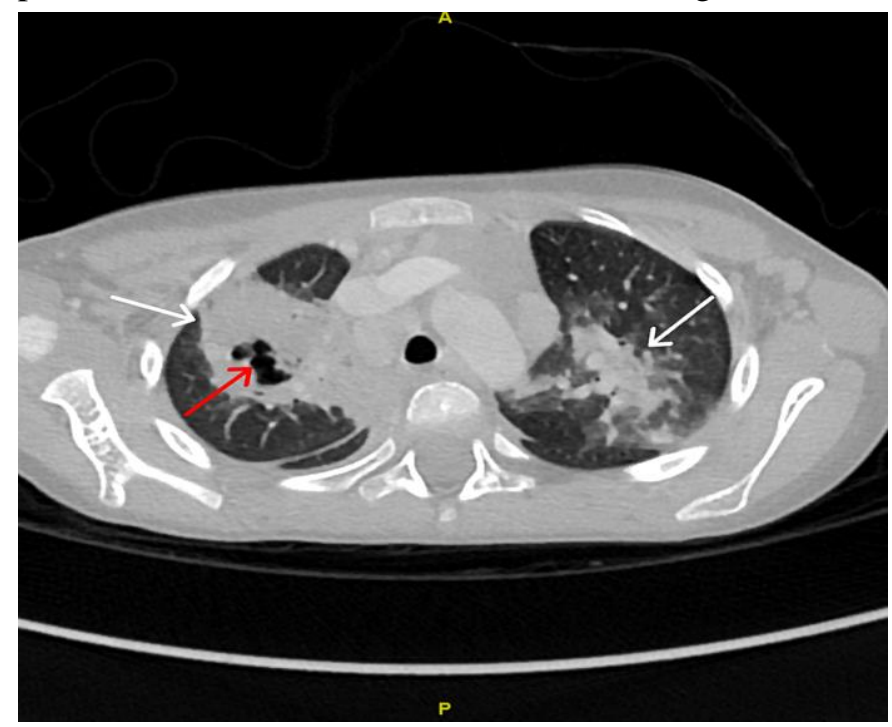

Figure (2): Contrast-enhanced chest computed tomography $(\mathrm{CT})$ confirmed bilateral pneumonic consolidation (white arrows) with cavitation (red arrow)
The rheumatology team requested further examinations, including tests for anti-nuclear antibodies, complements, and anti-neutrophil cytoplasmic autoantibodies. All test results were normal. On the eighth day after admission, the patient became afebrile, and inflammatory markers gradually began to decline. However, after three weeks of illness, the patient developed desquamation of skin on the hands and feet. As a result, the final diagnosis was confirmed as KD with GAS. Treatment with vancomycin and tazocin was sustained for a whole of three weeks, and subsequently the case was discharged after a follow-up cardiology examination. Echocardiography was repeated after six weeks of illness with no evidence of coronary dilatation.

\section{Ethical approval:}

The case report was approved by the research ethics committee of the General Pediatric Unit, King Saud University Medical City, Riyadh, Saudi Arabia. In addition, written consent was obtained from the parents of the case after explaining the objective of the case report.

\section{DISCUSSION}

In our case, the diagnosis of KD was established on the diagnostic principles established by means of Tomisaku Kawasaki in 1967. These criteria include persistent fever continued for five days or longer in addition to minimum four of the next five physical signs: cervical lymphadenopathy, bilateral non-purulent conjunctivitis, oral mucosal changes, polymorphous rash, and outer extremity deviations comprising erythema of palms or soles, edema of hands or feet, or periungual desquamation ${ }^{(5)}$. Lung involvement in $\mathrm{KD}$ is rare. A previous study of 602 children with KD showed that only $1.8 \%$ presented with mostly respiratory symptoms ${ }^{(6)}$. Atypical KD can manifest as bronchopneumonia, hydropneumothorax, and pleural effusion ${ }^{(7)}$. However, the exact cause of KD is still indefinite. Inflammatory cell penetration into vascular soft tissue causes obliteration of elastin and collagen fibers resulting in disruption of the structural honesty of the arterial wall, leading to dilatation and aneurysm formation ${ }^{(8,9)}$.

Infectious etiology is one of the theories that have been proposed based on resemblances among KD and additional pediatric communicable disorders. The illness frequently take place in clusters. In addition, a geographical wave-like blowout of disorder throughout epidemics has been detected, suggesting an infectious trigger ${ }^{(10)}$. The number of cases among family members tends to be higher than that of the incidence rate among general population. An incubation period of less than a week was observed in more than $50 \%$ of family cases ${ }^{(11)}$. In addition, clinical features of KD overlap with contagious illnesses, especially toxin-mediated diseases, 
such as scarlet fever as well as toxic shock syndrome. However, such illnesses typically lack ocular and articular engrossment, which is characteristic of KD. Many microorganisms have been linked to $\mathrm{KD}$ such as Streptococcus, Staphylococcus, Mycoplasma, EpsteinBarr virus, coronavirus, and parvovirus ${ }^{(12-16)}$.

Lately, a novel coronavirus, SARS-CoV-2, which causes COVID-19, was recognized as the reason of a group of pneumonia cases in Wuhan, China. In children, COVID-19 is usually mild. However, in some cases, the disease can be severe and present as toxic shock syndrome or KD. In children, this disorder is named multisystem inflammatory syndrome (MIS-C), and a previous report showed that $40 \%$ of MIS-C patients encountered the standards for comprehensive or incomplete $\mathrm{KD}$. Nevertheless, shock occurs more commonly in MIS-C than in classic $\mathrm{KD}$, besides complete lymphocyte and platelet counts inclined to be lesser in MIS-C than individuals in typical KD ${ }^{(17)}$.

In our case, GAS, which is usually observed in patients with KD, was diagnosed. According to a study conducted by Benseler et al. ${ }^{(18)}, 42$ of 134 patients diagnosed with $\mathrm{KD}$ had a confirmed infection most commonly associated with GAS pharyngitis. Patients with GAS exhibit a wide variety of clinical signs, ranging from slight skin/soft tissue contaminations and pharyngitis to additional severe conditions, like bacteremia, toxic shock syndrome, meningitis and also NF (Necrotizing Fasciitis). In contrast, pneumonia has very few clinical findings ${ }^{(4)}$. A noteworthy and rarely reported feature in our case was the combination of clinical findings of invasive pneumonia with desquamating skin rash due to GAS and KD.

Patients with pneumonia may have a higher risk of coronary artery abnormalities, most likely due to delay in diagnosing KD. Previously, there has been a report of a case wherein the patient had a history of pneumonia complicated by coronary artery involvement and a delay in the administration of IVIG, resulting in a poor response to antibiotics ${ }^{(19)}$.

\section{CONCLUSIONS}

A diagnosis of KD should be considered in patients with pneumonia who exhibit the characteristic symptoms of $\mathrm{KD}$, especially when a poor response to antibiotic treatment is observed.

\section{REFERENCES}
1. WHO
(2021):
Pneumonia.
(2021). https://www.who.int/news-room/fact- sheets/detail/pneumonia/.

2. McIntosh K (2002): Community-acquired pneumonia in children. N Engl J Med., 346: 429-437.
3. Pichichero M (1998): Group A beta-hemolytic streptococcal infections. Pediatr Rev., 19: 291-302.

4. Lamagni TL, Darenberg J, Luca-Harari B et al. (2008): Epidemiology of severe Streptococcus pyogenes disease in Europe. J Clin Microbiol., 46: 2359-2367.

5. Kawasaki T (1967): Acute febrile mucocutaneous syndrome with lymphoid involvement with specific desquamation of the fingers and toes in children. Arerugi., 16: 178.

6. Singh S, Gupta A, Jindal A et al. (2018): Pulmonary presentation of Kawasaki disease-A diagnostic challenge. Pediatr Pulmonol., 53: 103-107.

7. Arslanoglu Aydin E, Demir S, Aydin O et al. (2019): Pleural effusion as an atypical presentation of Kawasaki disease: a case report and review of the literature. Journal of medical case reports, 13 (1): 344.

8. Jennette J, Falk $\mathbf{R}$ (2007): The role of pathology in the diagnosis of systemic vasculitis. Clin Exp Rheumatol., 25: 52.

9. Naoe S, Takahashi $\mathrm{K}$, Masuda $\mathrm{H}$ et al. (1991): Kawasaki disease. With particular emphasis on arterial lesions. Acta Pathol Jpn., 41: 785.

10. Burns JC, Cayan DR, Tong G et al. (2005): Seasonality and temporal clustering of Kawasaki syndrome. Epidemiology, 16: 220.

11. Yanagawa $H$, Nakamura $Y$, Yashiro $M$ et al. (1988): A nationwide incidence survey of Kawasaki disease in 1985-1986 in. Japan. J Infect Dis., 158: 1296.

12. Lee $M$, Cha J, Ahn $H$ et al. (2011): Mycoplasma pneumoniae infection in patients with Kawasaki disease. Korean J Pediatr., 54: 123-127.

13. Matsubara K, Fukaya $T$ (2007): The role of superantigens of group A Streptococcus and Staphylococcus aureus in Kawasaki disease. Curr Opin Infect Dis., 20: 298-303.

14. Kikuta H, Sakiyama $\mathrm{Y}$, Matsumoto $\mathrm{S}$ et al. (1993): Detection of Epstein-Barr virus DNA in cardiac and aortic tissues from chronic, active EpsteinBarr virus infection associated with Kawasaki diseaselike coronary artery aneurysms. J Pediatr., 123: 90-92.

15. Esper F, Shapiro E, Weibel C (2005): Association between a novel human coronavirus and Kawasaki disease. J Infect Dis., 191: 499-502.

16. Holm J, Hansen L, Oxhoj H (1995): Kawasaki disease associated with parvovirus B19 infection. Eur J Pediatr., 154: 633-634.

17. Feldstein L, Rose E, Horwitz $\mathrm{S}$ et al. (2020): Multisystem Inflammatory Syndrome in U.S. Children and Adolescents. N Engl J Med., 383: 334346.

18. Benseler S, McCrindle B, Silverman $\mathrm{E}$ et al. (2005): Infections and Kawasaki disease: implications for coronary artery outcome. Pediatrics, 116: 760-766.

19. Arslanoglu E, Demir S (2019): Pleural effusion as an atypical presentation of Kawasaki disease: a case report and review of the literature. J Med Case Reports, 13: 344. 